\title{
Estimación del riesgo de fallo en el suministro de agua como ayuda a la planificación y gestión de recursos hídricos
}

\author{
Alex Avilés A. ${ }^{1,2}$, Abel Solera S. $^{3}$ \\ ${ }^{1}$ Grupo de Ciencias de la Tierra y del Ambiente, Dirección de Investigación, Universidad de Cuenca, \\ Av. 12 de Abril s/n, Cuenca, Ecuador. \\ ${ }^{2}$ Facultad de Ciencias Químicas, Escuela de Ingeniería Ambiental, Universidad de Cuenca, Av. 12 de \\ Abril s/n, Cuenca, Ecuador. \\ ${ }^{3}$ Instituto de Ingeniería del Agua y Medio Ambiente, Universidad Politécnica de Valencia, Camino de \\ vera s/n, Valencia 46022, España.
}

Autor para correspondencia: alex.aviles@ucuenca.edu.ec

Fecha de recepción: 5 de noviembre 2012 - Fecha de aceptación: 15 de enero 2013

\section{RESUMEN}

En este estudio modelos numéricos y escenarios estocásticos se utilizaron para analizar si sistemas de recursos hídricos en la cuenca del río Paute, bajo ciertas condiciones de infraestructura (embalses de almacenamiento), son capaces de satisfacer demandas de agua en los siguientes 30 años. Los análisis revelaron la necesidad de implementar un embalse con una capacidad de al menos de $21 \mathrm{Hm}^{3}$ en la subcuenca del río Tomebamba, mientras que en la subcuenca del río Pindilig no se requerirá ningún embalse. En caso de que el suministro de agua durante los periodos secos esté por debajo de la demanda de agua podría requerirse la aplicación de medidas de restricción en el uso del agua. Los resultados muestran claramente la utilidad del enfoque utilizado y su capacidad para generar alternativas en la oferta y demanda de agua en condiciones futuras. Como tal, el método será muy útil para los tomadores de decisiones en la definición de las políticas más adecuadas para la planificación y gestión del recurso hídrico.

Palabras clave: Recursos hídricos, modelación numérica, escenarios estocásticos, tomadores de decisiones, gestión y planificación.

\begin{abstract}
In this study numerical models and stochastic scenarios are used to verify if the water resource systems of the Paute River Basin under the given conditions of infrastructure (storage reservoirs) are capable of meeting the water demand in 30 years from today. The analysis revealed the need to implement a reservoir with a capacity of at least $21 \mathrm{Hm}^{3}$ in the Tomebamba subbasin, whereas the Pindilig subbasin does not require any storage reservoir. In case the water supply during dry periods ceases below the water demand the implementation of water use restriction measures might be required. The results clearly show the usefulness of used approach and its capacity in generating alternatives in water supply and demand for future conditions. As such the method will be very helpful to decision-makers in defining the most appropriate policies for the planning and management of the water resources.
\end{abstract}

Keywords: Water resources, numerical modeling, stochastic scenarios, decision-making, management, and planning.

\section{INTRODUCCIÓN}

Los sistemas de recursos hídricos son una mezcla de muchos aspectos ambientales, sociales, hidrológicos, económicos y administrativos, su administración requiere amplias e integradas toma de 
decisiones, que requieren información de las diferentes necesidades de los usuarios y los conflictivos intereses económicos y sociales por acceder al agua (Alemu y col., 2011). Variaciones espaciales y temporales en los componentes del sistema dan cuenta que son funciones de muchos factores estocásticos, es por esto que los análisis de los sistemas van más allá de los métodos convencionales deterministas (Huang y col., 2010). Durante la explotación de los sistemas de recursos hídricos, pueden darse inevitablemente situaciones en las que no sea posible suministrar de forma satisfactoria los usos establecidos (fallo del sistema, denominado también sequía operativa), esta situación puede minimizarse mediante una anticipación de la sequía en el sistema en las fases de planificación y explotación, minimizando los valores esperados de duración y magnitud de sequías, mediante la adopción de reglas de gestión (Sánchez y col., 2000).

Las políticas y reglas de operación de un sistema de recursos hídricos son herramientas fundamentales para la toma de decisiones durante la fase de explotación de un sistema, el uso de una regla u otra, o de ninguna regla, es determinante para el rendimiento del sistema (Solera, 2003). Debe destacarse que no existen reglas o estrategias de gestión universales para el manejo de los recursos hídricos; sin embargo, algunas reglas son extrapolables a sistemas con topologías y propósitos similares, proponiendo una amplia gama de experiencias en el manejo de sistemas complejos que pueden utilizarse como ejemplos para adoptar reglas para un sistema particular (Lund y Guzmán, 1999). Dolling (2001) y Ochoa (2002), hacen una descripción de algunas herramientas informáticas destinadas a resolver distintos problemas del área de la hidrología y de la gestión de los sistemas hídricos, en donde se incluyen modelos desarrollados para el diseño, planificación y explotación de un sistema hídrico, estos modelos tienen incorporados modelos matemáticos complejos de simulación y optimización para los distintos análisis en los sistemas de recursos hídricos, cuyo objetivo final es ayudar a los tomadores de decisiones para identificar políticas o reglas de operación en la planificación y gestión del agua. A continuación se detallan algunos modelos con la finalidad de mostrar las ventajas de utilización de unos u otros.

REZES (Simonovic y Savic, 1989) es un programa diseñado como herramienta para la asistencia al análisis de embalses (dimensionamiento, planificación de operación, operación a tiempo real), en donde están incluidos métodos de optimización (programación determinística, programación estocástica). AQUARIUS (Diaz y Brown, 1997) es un sistema orientado a la distribución temporal y espacial de agua entre los usuarios en una cuenca hidrográfica, utilizando programación no lineal. HEC-5 (Hydrologic Engineering Center, 1998) es un modelo que asigna el agua a las demandas mediante el balance hídrico de una cuenca, teniendo en cuenta las reglas de operación dadas por el usuario, además admite la consideración de múltiples propósitos. MODSIM (Labadie y col., 2000) tiene una estructura de trabajo basado en algoritmos de optimización de programación dinámica, la función objetivo está dada en términos de costos, y los repartos y almacenamientos de agua se hacen según una escala de prioridades dada por el modelador. WEAP (SEI, 2001) es un modelo enfocado a la evaluación y planificación hidrológica de cuencas; dicho modelo trabaja con algoritmos de balance hídrico mediante redes de flujo, en las que tiene la posibilidad de considerar como elementos físicos del sistema, embalses, canales, tramos de ríos, tuberías y demandas, etc. AQUATOOL (Andreu y col., 1996) es un software para el diseño y desarrollo de sistemas de soporte a la decisión (SSD) para gestores de recursos hídricos, que permite, entre otras cosas diseñar e introducir gráficamente la configuración de un sistema de recursos hídricos y sus características físicas y condiciones de gestión, para su posterior optimización y simulación.

Como se puede ver en el párrafo anterior, existe una amplia variedad de modelos que resuelven problemas y necesidades de gestión y planificación de agua mediante modelos de simulación y optimización; no obstante, el uso de uno u otro modelo va a depender de los objetivos y recursos disponibles de cada estudio. En el presente documento se utiliza dos modelos SIMGES (Andreu y col., 2010) y SIMRISK (Andreu y col., 2003) que están integrados en el SSD AQUATOOL, son modelos amigables con el usuario y su utilización no representa costos computacionales excesivos. SIMGES realiza la simulación de sistemas de recursos hídricos utilizando una red de flujo interna, basándose en el principio de conservación de masa; el modelo optimiza la red interna con un algoritmo lineal, dando como resultado la asignación de agua para los distintos usos, según un orden de prioridades de las demandas previamente establecido. En cambio SIMRISK es un modelo muy útil y eficaz para la simulación con escenarios estocásticos, el modelo proporciona al decisor de herramientas para la 
gestión, como la determinación de las probabilidades de fallos en los sistemas de recursos hídricos y la evaluación de los riesgos de las decisiones en el sistema y las medidas de mitigación según el riesgo admisible impuesto.

Con estos antecedentes, el presente trabajo pretende estimar el riesgo de fallo en el suministro de agua para generar medidas adecuadas en la oferta y la demanda de agua en los próximos 30 años en dos subcuencas del río Paute (Tomebamba y Pindilig) con diferente régimen de precipitación. Para lo cual se recurrió a métodos numéricos de simulación utilizando escenarios estocásticos como entrada.

\section{MATERIALES Y MÉTODOS}

\subsection{Materiales}

La Figura 1 muestra un mapa de la cuenca del río Paute, la misma que tiene gran importancia por sus abastecimientos urbanos e hidroeléctricos; sin embargo, adolece de una ausencia de infraestructuras de regulación en la mayoría de subcuencas, esto sumado a la variabilidad climática en la cuenca que conlleva a que existan épocas de sequía severas que afectan al suministro de agua de las demandas consuntivas, no consuntivas y ambientales.

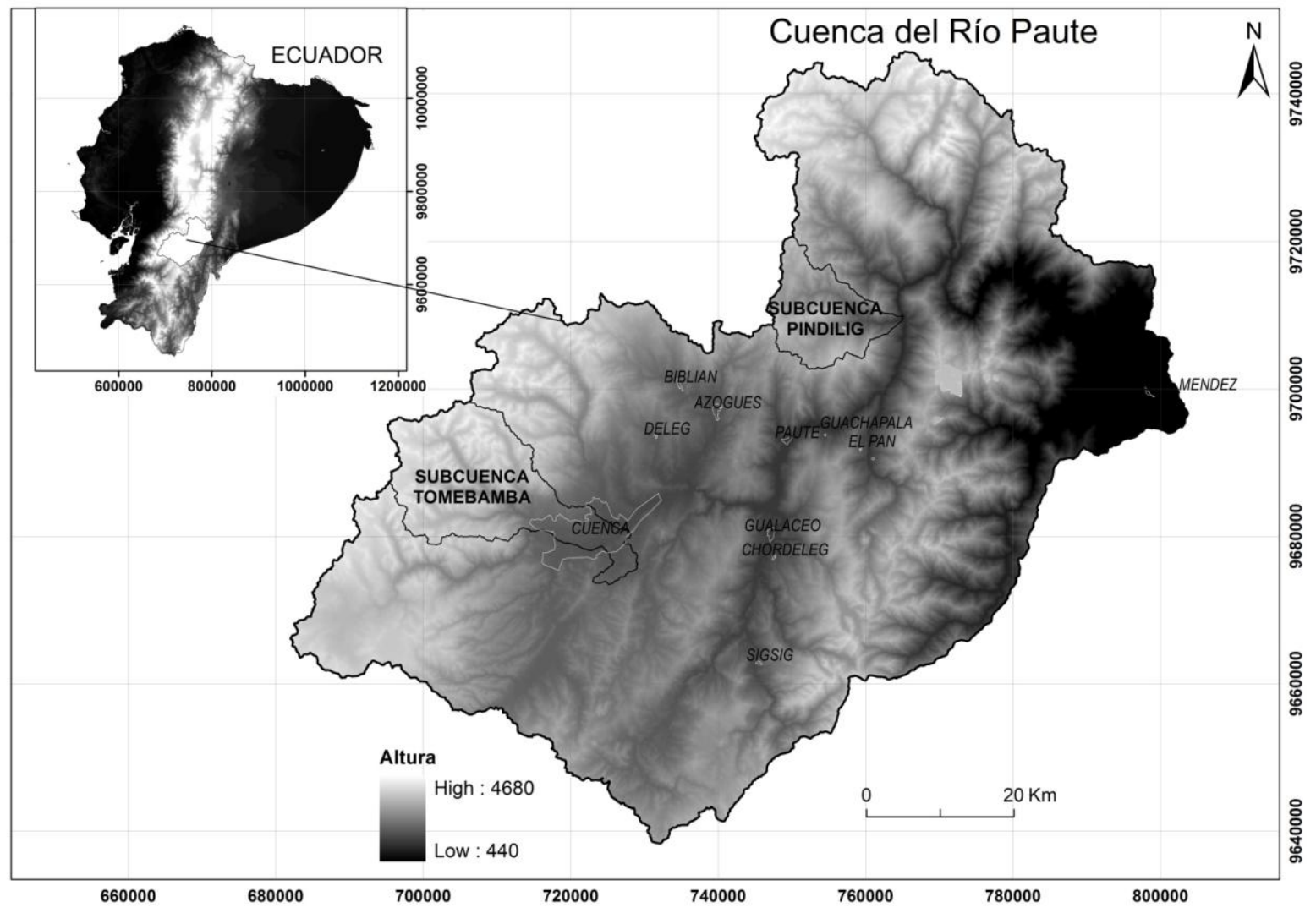

Figura 1. Ubicación del ámbito de estudio.

Para los modelos numéricos estocásticos para la síntesis de aportaciones se utilizó registros históricos de caudales mensuales de estaciones hidrológicas ubicadas al final de las áreas consideradas naturales de las subcuencas de estudio, es decir, donde no existe una explotación considerable del recurso hídrico. La estación hidrológica Matadero en Sayausí (ubicada en la subcuenca del río Tomebamba) y la estación hidrológica Dudas en Pindilig (ubicada en la subcuenca del río Pindilig) fueron las escogidas para extraer la información de aportaciones mensuales para aplicar los distintos 
modelos. Estas series también sirvieron de datos de entrada para simular el escenario actual en el modelo SIMGES conjuntamente con las demandas de agua actuales.

Para cuantificar las demandas actuales de agua se utilizaron las bases de datos de la Secretaría Nacional del Agua (SENAGUA) y de la Empresa Municipal de Agua Potable de la Ciudad de Cuenca (ETAPA), en donde está expuesta información de los diferentes usos de agua. Estas demandas fueron agrupadas según una prioridad en el uso del recurso (primero el agua para consumo doméstico, luego el agua para riego y al final los otros usos del agua) y según el peso que tienen las demandas que utilizan un mayor volumen de agua, con el propósito de simplificar los esquemas de los sistemas de recursos hídricos construidos para los modelos de simulación. Para atender los requerimientos ambientales de los ecosistemas fluviales, se consideró un caudal ecológico equivalente al $10 \%$ de los caudales medios mensuales. El cálculo de la demanda futura se realizó de una manera sencilla, considerando proyecciones lineales de la población, hectáreas de riego, semovientes, etc., y posibles dotaciones futuras según las tendencias potenciales de uso del agua en las zonas de estudio. La demanda futura y las series sintéticas de aportaciones fueron la información de entrada para el modelo SIMRISK.

\subsection{Modelos estocásticos de series de tiempo}

Los modelos estocásticos se fundamentan principalmente en el máximo aprovechamiento de la información de la serie histórica de una variable determinada, es decir reproducir las estadísticas históricas de las series de tiempo; el concepto clave en este tipo de modelos es la dependencia entre los datos, su aplicación presenta algunas ventajas, por ejemplo tiene mucha flexibilidad para trabajar en diferentes escalas, además son de fácil entendimiento y no requieren altos requerimientos computacionales.

En el presente estudio se aplicaron modelos autoregresivos de media móvil (ARMA) y modelos autoregresivos de media móvil periódicos (PARMA), con diferentes órdenes (p,q), se utilizó como ayuda el software SAMS (Sveinsson y col., 2007), cuyo objetivo fue conseguir un conjunto de modelos que sean capaces de reproducir las propiedades estadísticas históricas de los datos (estadísticos básicos, sequía y almacenamiento) y esencialmente las suposiciones claves de los residuos como la normalidad y la independencia. Finalmente, se escogió los mejores modelos con los menores valores del criterio de información de Akaike (1974) corregido (AICC) (Hurvich y Tsai, 1989), el mismo que se basa en una penalización de los modelos con mayor número de parámetros. Estos modelos sirvieron para la generación de series sintéticas anuales (utilizadas para la implementación de infraestructura para la regulación del agua) y la generación de series sintéticas mensuales (utilizadas para la construcción de escenarios futuros para la simulación de la gestión de la infraestructura planificada).

\subsection{Simulación de los sistemas de recursos hídricos utilizando SIMGES}

El proceso de simulación realiza una optimización de una red de flujo conservativa, mediante el cumplimiento de una función objetivo (satisfacción de las demandas, operación de embalses, etc.) sujeta a las restricciones de conservación de masa (continuidad) y los límites físicos de transporte de flujo en conducciones (ríos, quebradas, etc.) y capacidades de embalses (reservas) y otros elementos si los hubiere.

A partir de las series históricas de caudales y la agrupación de las demandas del agua, se construyeron diseños de cada uno de los sistemas de recursos hídricos para cada subcuenca, un ejemplo del esquema construido para la subcuenca del río Pindilig se muestra en la Figura 2. Una vez diseñados los esquemas, mediante el modelo SIMGES, se procedió a simular el escenario actual (1979-2009) mediante la introducción de la información de la serie de aportaciones mensuales históricas y la demanda actual, luego el escenario futuro (2010-2040) con la introducción de la demanda futura y la información de la serie de aportaciones mensuales históricas, suponiendo que el régimen natural de flujo de las subcuencas no variará con el tiempo. En caso de un incumplimiento en la satisfacción de las demandas se planificó la implementación de un embalse con una capacidad ideal para la satisfacción total de las demandas; sin embargo, esta capacidad ideal supone que no existirán restricciones físicas, económicas, sociales y ambientales en la construcción de la infraestructura, es por 
esto que se probó la implementación de un embalse con una capacidad menor, dando como resultado una insatisfacción de las demandas y el inicio de una gestión de los sistemas con un riesgo de desabastecimiento de agua.

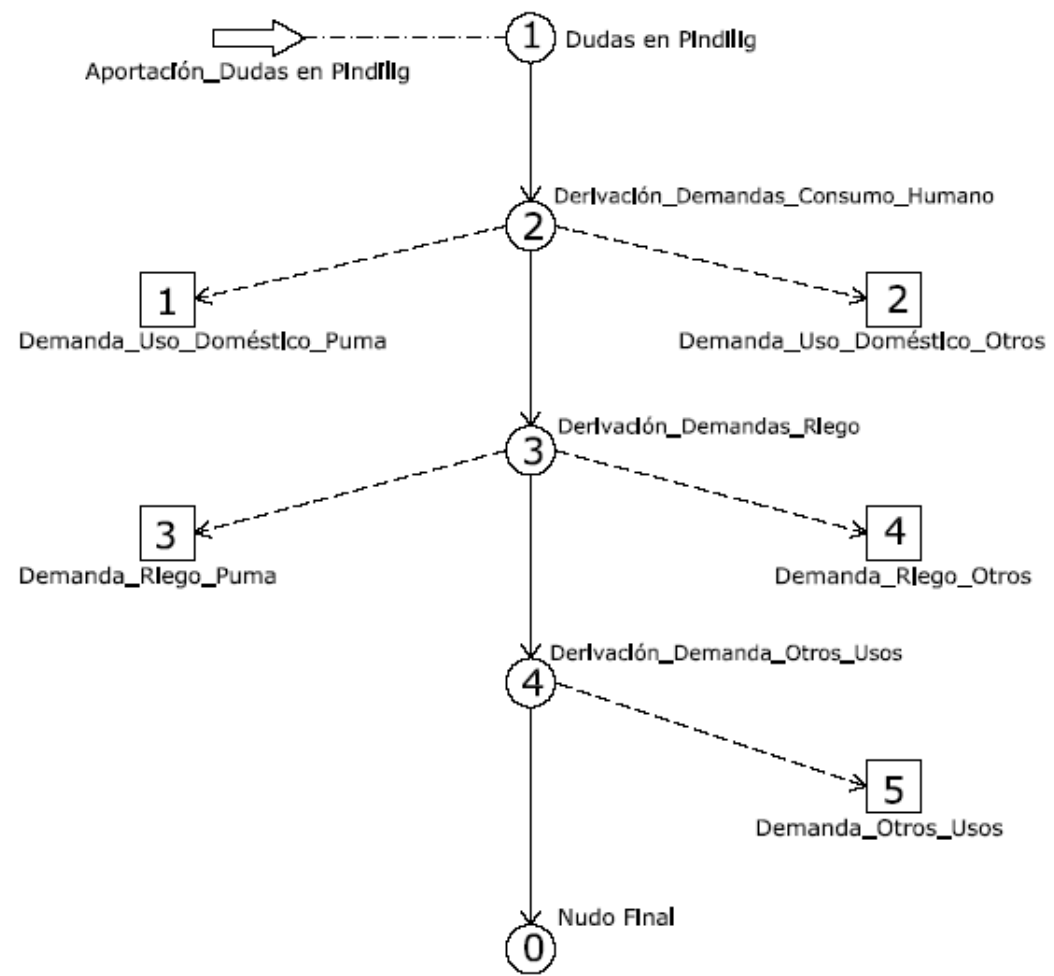

Figura 2. Esquema del sistema de recursos hídricos en la subcuenca del río Pindilig.

\subsection{Simulación de la gestión con escenarios estocásticos utilizando SIMRISK}

Con la ayuda de SIMRISK se simuló la gestión de 100 escenarios estocásticos, creados por cada serie sintética mensual, tomando en cuenta la implementación de un embalse con una capacidad no ideal, luego se verificó de una manera probabilística la satisfacción de las demandas, determinando el estado del sistema mediante la evaluación de las probabilidades de fallo mensual y la magnitud del déficit en el suministro (dividido en cuatro niveles). Por ejemplo, el nivel 1 (n1) es la situación más grave, quiere decir que el déficit supera el $75 \%$ de la demanda, es decir, el suministro se ubica entre el 0 y $25 \%$ del valor de la demanda; el nivel 2 (n2) quiere decir que el suministro se ubica entre el 25 y $50 \%$ del valor de la demanda; el nivel 3 (n3) quiere decir que el suministro se ubica entre el 50 y $75 \%$ del valor de la demanda; y el nivel 4 (n4) es la situación menos grave, quiere decir que el suministro se ubica entre el 75 y $100 \%$. Todo este marco probabilístico sirvió de ayuda para generar alternativas de gestión de las demandas para que el riesgo de fallo sea el menor posible.

\section{RESULTADOS}

\subsection{Modelos estocásticos de series de tiempo}

Según el AICC los mejores modelos ARMA para la subcuenca del río Tomebamba fueron el $\operatorname{ARMA}(1,1)$ para la escala anual y el AR(1) para la escala mensual; y para la subcuenca del río Pindilig fueron el MA(3) para la escala anual y mensual. De igual forma los mejores modelos PARMA mensuales según el AICC se muestran sombreados en la Tabla 1 para la subcuenca del río Tomebamba y en la Tabla 2 para la subcuenca del río Pindilig. Estos modelos sirvieron para la generación de series sintéticas anuales y mensuales, cuyos estadísticos resultantes fueron similares con 
los estadísticos de las series históricas. Se escogió trabajar con los modelos PARMA a escala mensual debido a que los estadísticos de sequía y almacenamiento de las series generadas tuvieron una mejor representación de los estadísticos de la muestra histórica.

Tabla 1. Valores AICC de los modelos PARMA en la subcuenca del río Tomebamba.

\begin{tabular}{cccc}
\hline Mes & PARMA(1,0) & PARMA(1,1) & PARMA $(1,2)$ \\
\hline ENE & 31.39 & 33.06 & 34.24 \\
FEB & 26.97 & 28.57 & 31.75 \\
MA & 28.21 & 29.58 & 31.41 \\
ABR & 22.30 & 22.08 & 25.42 \\
MA & 28.29 & 28.45 & 30.81 \\
JUN & 33.91 & 36.36 & 37.79 \\
JUL & 33.99 & 32.58 & 33.72 \\
AG & 30.32 & 20.82 & 23.30 \\
SEP & 21.75 & 23.79 & 26.46 \\
OCT & 33.18 & 35.41 & 31.66 \\
NO & 35.43 & 37.87 & 36.20 \\
DIC & 24.73 & 22.68 & 27.27 \\
\hline
\end{tabular}

Tabla 2. Valores AICC de los modelos PARMA en la subcuenca del río Pindilig.

\begin{tabular}{cccccc}
\hline Tabl & PARMA(1,0) & PARMA(3,0) & PARMA(0,2) & PARMA(0,3) & PARMA(1,1) \\
\hline ENE & 6.87 & 11.72 & 20.77 & 6.59 & 9.29 \\
FEB & 1.43 & 3.57 & 20.10 & -1.60 & 3.88 \\
MA & 14.68 & 18.48 & 14.57 & 23.77 & 16.47 \\
AB & 21.26 & 25.72 & 25.46 & 22.40 & 23.32 \\
MA & 19.63 & 14.12 & 31.02 & 20.19 & 15.29 \\
JUN & 20.96 & 24.75 & 24.58 & 26.27 & 22.57 \\
JUL & 15.35 & 20.14 & 22.46 & 22.80 & 17.70 \\
AG & 11.55 & 13.64 & 20.73 & 18.25 & 11.15 \\
SEP & -2.61 & 1.83 & 7.17 & 10.13 & -0.16 \\
OCT & 19.24 & 23.80 & 26.39 & 24.60 & 21.38 \\
NO & 5.19 & 8.38 & 14.96 & 15.49 & 6.56 \\
DIC & -11.76 & -13.67 & -0.49 & -3.04 & -15.33 \\
\hline
\end{tabular}

\subsection{Simulación de los sistemas de recursos hídricos utilizando SIMGES}

Los resultados de la simulación de los escenarios actual y futuro en la subcuenca del río Tomebamba revelaron que todas las demandas tienen déficits en algunos meses (incluyendo la demanda ambiental), además no cumplían con la garantía (es decir, no existía satisfacción de las demandas). Por lo tanto, se intentó incluir una infraestructura de regulación virtual (embalse), de tal manera que las garantías de las demandas sean cumplidas. En cambio, en la subcuenca del río Pindilig los resultados de la simulación de los escenarios actual y futuro revelaron el cumplimiento total de las garantías de las demandas (incluida la demanda ambiental); por lo tanto, en este sistema no haría falta una infraestructura de regulación al menos en los siguientes 30 años (con la información disponible y estimada).

Con las 100 series sintéticas anuales generadas en la estación de caudales de la subcuenca del río Tomebamba y con un nivel de demanda igual a la demanda anual total futura en la subcuenca, se calculó 100 valores de capacidad de almacenamiento, donde 40 valores fueron iguales a cero y 60 valores diferentes de cero. Con un valor medio de los valores no nulos de capacidad de 
almacenamiento que corresponde a un valor aproximadamente igual a $21 \mathrm{Hm}^{3}$ se procedió a simular el sistema de recursos hídricos de la subcuenca del Tomebamba en el escenario futuro, dando como resultado un cumplimiento total de las garantías de las demandas mensuales y anuales. También hay que indicar que la demanda ambiental cumplió satisfactoriamente en todos los meses.

Probando bajar la capacidad del embalse a la mitad aproximadamente $\left(10 \mathrm{Hm}^{3}\right)$, suponiendo que esta capacidad será factible según unas restricciones físicas, ambientales, sociales y económicas, que se podrían presentar en la construcción de una infraestructura de tal magnitud, se incumpliría nuevamente las garantías de las demandas y daría inicio a una gestión futura del sistema, con el embalse factible planificado, con escenarios estocásticos y con enfoque de riesgo de desabastecimiento de agua a las demandas.

\subsection{Simulación de la gestión con escenarios estocásticos utilizando SIMRISK}

Para que sean más entendibles los resultados se realizó un promedio mensual de los valores de probabilidad de fallo para cada nivel de suministro (n1, n2, n3 y n4) y para cada mes en el periodo de simulación, esto es, por cada mes del año hidrológico se obtendrá una probabilidad de fallo de la satisfacción total de las demandas. Por ejemplo, el mes de Febrero es el más desfavorable para el suministro de agua para la ciudad de Cuenca y tiene una probabilidad de fallo total de $6.33 \%$ (Figura 3 ). De igual forma, las demandas de riego presentan el mes de Febrero como el más desfavorable para el suministro de agua, con una magnitud de probabilidad de fallo total de $8.47 \%$ y una probabilidad de fallo en el nivel 1 de $6.90 \%$, es decir, en el nivel con un déficit de $75 \%$ de la demanda (Figura 4).

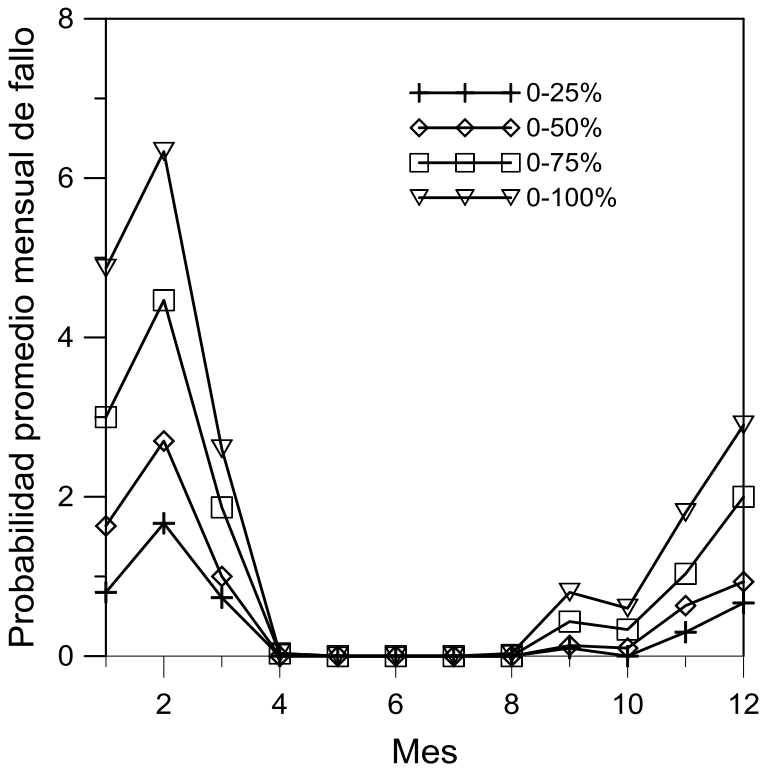

Figura 3. Probabilidad promedio mensual de fallo de la demanda futura de la ciudad de Cuenca.

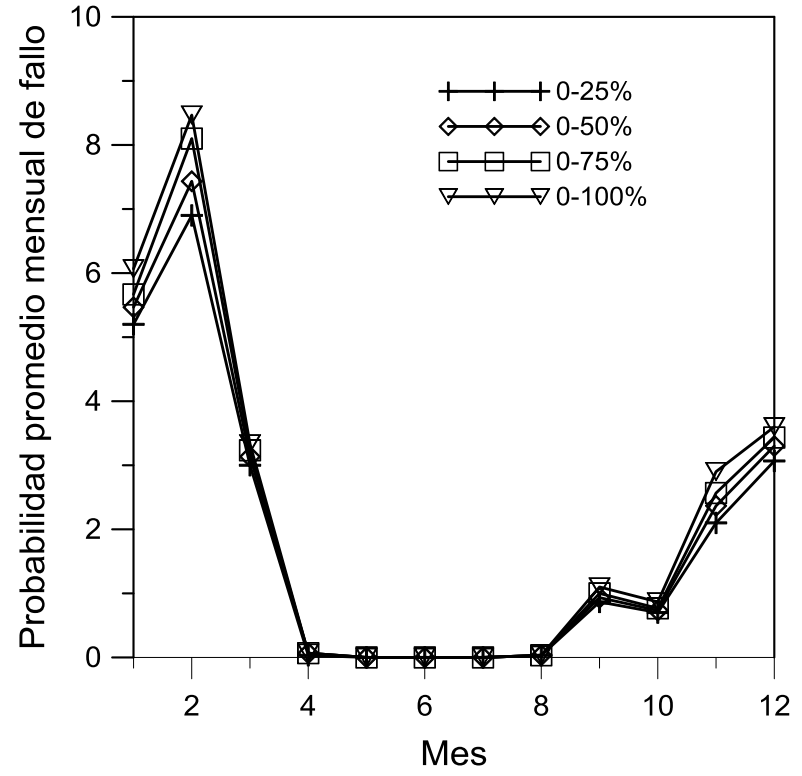

Figura 4. Probabilidad promedio mensual de fallo de las demandas futuras de riego.

Podría interesar para la gestión futura del sistema bajar el valor de estimación de probabilidad de fallo en las demandas, sobre todo de las prioritarias, por lo tanto se analizó la posibilidad de implementar una restricción en los usos del agua destinadas para el riego, abrevaderos de ganado y las piscícolas, de tal manera de bajar la probabilidad de fallo en el suministro para las demandas de consumo humano, especialmente de la ciudad de Cuenca. Entonces se realizó la simulación múltiple con una restricción del $50 \%$ de las demandas con menor prioridad, manteniendo fija la capacidad factible del embalse $\left(10 \mathrm{Hm}^{3}\right)$. Este procedimiento dio como resultado la disminución de los valores promedios mensuales de probabilidad total, el mes de febrero considerado el mes más crítico bajó de 6.33\% a 3.87 (Figura 5), demostrando la utilidad de la metodología para disminuir el riesgo de fallo en el suministro de agua, principalmente de las demandas de agua más prioritarias. 


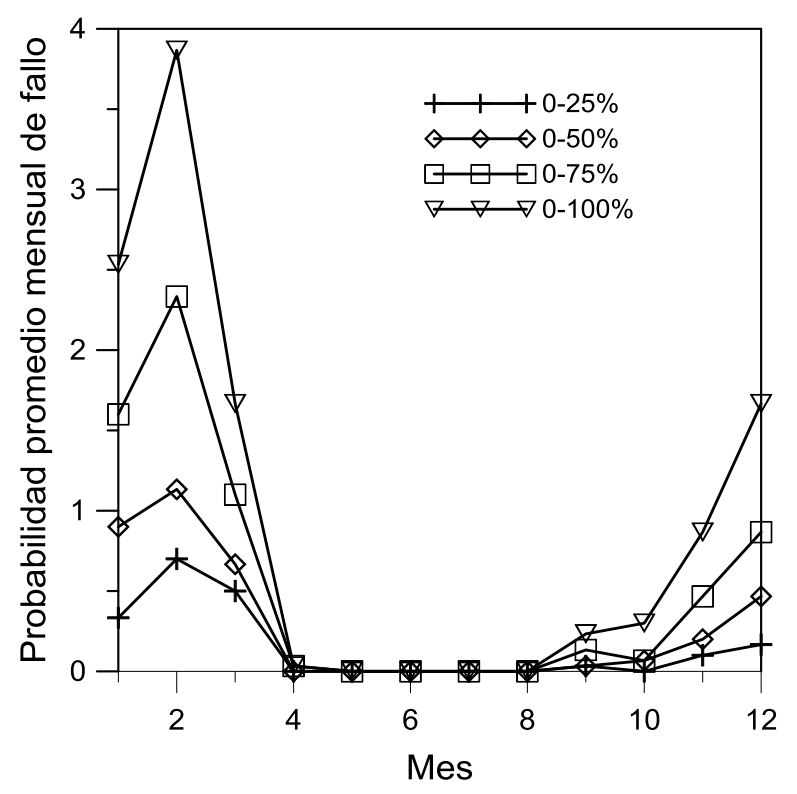

Figura 5. Probabilidad promedio mensual de fallo de la demanda futura de la ciudad de Cuenca en el escenario con restricciones a las demandas menos prioritarias.

\section{CONCLUSIONES}

La metodología expuesta en el presente artículo proporciona la posibilidad de generar alternativas en la oferta y demanda de agua, muy necesarias para una planificación y gestión de sistemas de recursos hídricos en cuencas deficitarias de agua. Esta metodología podría ser una herramienta eficaz para inferir reglas y políticas del uso de agua en cuencas hidrográficas a nivel local, regional y/o nacional. Además, la introducción de reglas de gestión de sistemas de recursos hídricos con una introducción del riesgo asociado a la decisión, brinda a los gestores seguridad y confianza de contemplar los sistemas de manera global, así como también a cada uno de sus componentes.

La aplicación de la metodología con la información disponible y estimada, muestra que existirán sistemas de recursos hídricos con una inminente necesidad de implementación de infraestructuras de regulación del agua para satisfacer a las demandas de agua, como el sistema de la subcuenca del río Tomebamba, mientras el sistema de la subcuenca del río Pindilig no requiere de embalses al menos en los próximos 30 años; sin embargo, no hay que descartar esta posibilidad, dado que puedan surgir en el futuro nuevas demandas que no fueron consideradas en este estudio. Asimismo, existirán situaciones en donde no se puedan implementar embalses de gran magnitud, dando como resultado la necesidad de una gestión de sistemas de recursos hídricos con un riego de desabastecimiento de agua.

Los resultados en el sistema de la subcuenca del río Tomebamba muestran una disminución en el riesgo de desabastecimiento de agua en la demanda de consumo humano de la ciudad de Cuenca cuando se toma la decisión de restringir el suministro de agua a la mitad de las demandas con una prioridad menor (riego, abrevaderos de ganado, hidroeléctricas y piscícolas). Esta regla de gestión planificada supone un beneficio para las demandas prioritarias, aunque supone un costo en las demandas de riego y otros usos, dado que no tendrán el suministro total para sus fines. No obstante, se pudo también poner restricciones de pequeña magnitud a las demandas prioritarias y así disminuir restricciones muy severas en las demandas menos prioritarias, pero esto ya dependerá de la toma de decisiones del gestor del sistema. Hacer notar que se pudo haber tomado como alternativa de gestión, la implementación de un embalse con una capacidad ideal (satisfacción total de las demandas), pero la capacidad de almacenamiento se ha pretendido limitarla para mostrar la utilidad de una metodología de gestión de sistemas de recursos hídricos cuando existe riesgo de desabastecimiento de agua.

En la aplicación de los casos de estudios se han extraído algunas enseñanzas, por ejemplo los resultados de la planificación de la gestión en la subcuenca del río Tomebamba mostraron que las 
situaciones más graves se producen en el mes de febrero, por lo cual este mes es de suma importancia para la gestión mensual futura de este sistema de recursos hídricos. Por otro lado, las reglas de gestión planificadas en la subcuenca del río Tomebamba deberán ser revisadas y perfeccionadas cuando el sistema esté en la fase de explotación. En esta fase se podría realizar una gestión del sistema en periodos muy cortos para la toma de decisiones, explorando modelos para predicción de caudales a corto plazo.

\section{AGRADECIMIENTOS}

El autor agradece al Profesor Jan Feyen por sus constructivas y valiosas sugerencias, las cuales mejoraron la estructura de este artículo.

\section{BIBLIOGRAFÍA}

Akaike, H., 1974. A new look at the statistical model identification. IEEE Transactions on Automatic Control, AS19 (6), 716-723.

Alemu E.T., R.N. Palmer, A. Polebitski, B. Meaker, 2011. Decision support system for optimizing reservoir operations using ensemble streamflow predictions. J. Water Res. Pl.-ASCE, 237(1), 7282.

Andreu, J., J. Capilla, E. Sanchís, 1996. AQUATOOL, a generalized decision-support system for water-resources planning and operational management. J. Hydrol., 177(3-4), 269-291.

Andreu, J., A. Solera, J. Paredes, 2003. Sistema de estimación de riesgos en la gestión de sequías. In: Jornadas sobre sistemas de ayuda a la decisión ante problemas hidrológicos e hidráulicos en tiempo real. CEDEX, Madrid, España.

Andreu, J., A. Solera, J. Capilla, J. Ferrer, 2010. SIMGES Simulation Model of Water Resource Management including Conjunctive Use. Version 3.02. User manual. Polytechnic University of Valencia, España, 100 pp. Descargado de http://www.upv.es/aquatool/docs/ManSIMGESI.pdf en septiembre 2012.

Diaz, G.E., T. Brown, 1997. AQUARIUS: A modeling system for river basin water allocation. General Technical Report RM-GTR-299, USDA Forest Service, Washington D.C., USA.

Dolling, O.R., 2001. Sistema de apoyo a la gestión integral de cuencas hidrográficas. Disertación Doctoral Ingeniero de Caminos, Canales y Puertos, Pontificia Universidad Católica de Chile, Santiago, Chile, $238 \mathrm{pp}$.

Huang, Y., Y.P. Li, X. Chen, A.M. Bao, M. Zhou, 2010. Simulation-based optimization method for water resources management in Tarim River Basin, China. Energ. Policy, 2, 1451-1460.

Hurvich, C.M., C.L. Tsai, 1989. Regression and time series model selection in small samples. Biometrika, 76(2), 297-307.

Hydrologic Engineering Center, 1998. HEC-5: Simulation of flood control and conservation systems, Version 8.0. Department of the Army Corps of Engineers, Davis, CA, USA. Descargado de http://www.hec.usace.army.mil/software/legacysoftware/hec5/hec5.htm en septiembre 2012.

Labadie, J.W., M.L. Baldo, R. Larson, 2000. MODSIM: Decision Support System for River Basin Management: Documentation and User Manual, Colorado State University and U.S. Bureau of Reclamation, Ft Collins, CO, USA.

Lund, J.R., J. Guzman, 1999. Some derived operating rules for reservoirs in series or in parallel. $J$. Water Res. Pl.-ASCE, 125(3), 143-153.

Ochoa, J.C., 2002. Modelo estocástico de redes neuronales para la síntesis de caudales aplicados a la gestión probabilística de sequías. Tesis Doctoral. Escuela Técnica Superior de Ingenieros de 
Caminos Canales y Puertos, Departamento de Ingeniería Hidráulica y Medio Ambiente, Universidad Politécnica de Valencia, Valencia, España, 205 pp.

Sánchez, S.T., J. Andreu, A. Solera, 2000. Gestión de recursos hídricos con decisiones basadas en estimación del riesgo. Publicaciones Universidad Politécnica de Valencia, Valencia, España, 158 pp.

SEI Stockholm Environment Institute, 2001. WEAP: Water Evaluation and Planning System.

Stockholm Environment Institute. Descargado de http://www.weap21.org/ en septiembre 2012.

Simonovic, S.P., D. Savic, 1989. Intelligent Decision Support and Reservoir management and operations. J. Comput. Civ. Eng., 3(4), 367-385.

Solera, A., 2003. Herramientas y métodos para la ayuda a la decisión en la gestión sistemática de recursos hídricos. Aplicación a las cuencas de los ríos Tajo y Júcar. Tesis Doctoral. Escuela Técnica Superior de Ingenieros de Caminos, Canales y Puertos, Departamento de Ingeniería Hidráulica y Medio Ambiente, Universidad Politécnica de Valencia, Valencia, España, 278 pp.

Sveinsson, O.G.B., J.D. Salas, W.L. Lane, D.K. Frevert, 2007. Stochastic analysis modeling and simulation (SAMS). User's Manual. Version 2007. Computing Hydrology Laboratory, Department of Civil and Environmental Engineering, Colorado State University, Fort Collins, $C O$, USA, $123 \mathrm{pp}$. 